\title{
Four powerful concepts to maximize healing
}

\section{Opinion}

\section{Patients, Are You Getting the Best Treatment from Your Healthcare Professional?}

Whether doctors and patients like it or not, there is a mind-body interaction. Healthcare professionals can leverage this interaction for enhanced healing.

As a healthcare professional, please consider these concepts to improve total diagnosis and treatment:

1. Knowledge and Medical Skills

2. Bedside Manner

3. Maximize the Placebo Effect

4. Minimize the Nocebo Effect

Knowledge and Medical Skills are taught very well in medical schools. Little need be said on this concept. It is the basis of many medical practices.

Bedside Manner is becoming more important as many practices are becoming consumer driven. Perhaps some healthcare professional have inherited good bedside manner. Others might want to learn. Some medical schools are combining "people skills" along with the usual curriculum. For those who want to learn more, some references are listed at the end of this article. In addition, here are good Internet search strings for even more information:

1. Listening-skills (patient OR doctor OR nurse)

2. Body-language (patient OR doctor OR nurse)

3. (Listening-skills OR body-language) tutorial

4. (Listening-skills body-language) tutorial

Most healthcare professionals do not consider Placebo/Nocebo Effects, though these effects are as powerful in many cases as medicine and treatment. Again, good techniques can be learned. General references are at the end of this article. To find more information on the Internet, use these search strings:

1. placebo-(effect or response) (words or wording or ritual or enhance or maximize) (patient or doctor or nurse or physician)

2. nocebo-(effect OR response) (words or wording or ritual or minimize or diminish) (patient or doctor or nurse or physician)

Use of these four concepts is somewhat of a judgment call by the practitioner. Some patients want only the facts and expertise. Some patients respond more to Bedside Manner. Some respond to Placebo/ Nocebo whereas some do not.

Patient-Doctor communication is bilateral. As people-skills are learned and practiced, doctors can pick up the patient's body language combined with listening to them. From that, the doctor or nurse can decide how to adjust his or her own words, body language, and actions. Maybe someday, there will be "Emotional Stress Indicators" on patients to help the doctor decide whether they are helping or
Volume 9 Issue 5 - 2018

Tom Garz

Writer and Inventor,TG Ideas LLC, USA

Correspondence: Tom Garz, Writer and Inventor,TG Ideas LLC, USA, Email tgidess@gmail.com

Received: May 28, 2018 | Published: October 15, 2018

hurting the patient. Medical practice is evolving. As much as there is hope for patients, there is hope for doctors and nurses willing to consider and learn new concepts and skills.

\section{Takeaways:}

1. Patient-Doctor communication is becoming more and more important, as healthcare becomes more consumer-driven.

2. Bedside Manner, Placebo Effect, and Nocebo Effect can all be leveraged for enhanced and sustained healing. The progressive and caring healthcare professionals can learn all these. As a patient, find a practitioner that fits you as a person. Ask around, explaining what is important to you, as a health care consumer. Give each practitioner a fair chance, but do your part to communicate your needs and wants. Healthcare professionals are not "Mind-Readers". ${ }^{1-12}$

\section{Acknowledgements}

None.

\section{Conflict of interest}

The author declares that there is no conflict of interest.

\section{References}

1. Bedside Manner.

2. Tara Parker-Pope. Can Better Bedside Manner Be Taught? Springer. 2009.

3. Person A, Finch L. Bedside Manner: Concept Analysis and Impact on Advanced Nursing Practice. Journal of Advanced Nursing Practice. 2008;10(1).

4. Travaline JM, Ruchinskas R, D’Alonzo GE. Patient-Physician Communication: Why and How. $J$ Am Osteopath Assoc. 2005;105(1):13-18.

5. David Watts. Bedside Manners: One Doctor's Reflections on the Oddly Intimate Encounters between Patient and Healer. Broadway Books; 2006. $306 \mathrm{p}$.

6. Sandra P Thomas. Listening to Patients: A Phenomenological Approach to Nursing Research and Practice. Springer Publishing Company; 2004. $312 \mathrm{p}$.

7. Howard Spiro. The Power of Hope: A Doctor's Perspective by Howard Spiro. Yale University Press; 1998. 314 p. 
8. Anne Harrington. The Placebo Effect: An Interdisciplinary Exploration. Harvard University Press; 1999. 272 p.

9. Program in Placebo Studies and the Therapeutic Encounter hosted. Beth Israel Deaconess Medical Center. 2017.

10. Yager J. Specific Components of Bedside Manner in the General Hospital
Psychiatric Consultation: 12 Concrete Suggestions. Psychosomatics. 1989;30(2):209-212.

11. As a Healthcare Professional, Are You Checking All the Vital Signs to Evaluate Total Patient Condition? 2017.

12. Medical Monitor with an Emotional Stress And/or Pain Indicator. 2018. 